\title{
APORTE DE LAS HERRAMIENTAS TERMINOLÓGICAS $Y$ DOCUMENTALES AL TRABAJO SOCIAL
}

\author{
Jenny Betsaida Cuervo Caro ${ }^{1}$
}

\section{Introducción}

$\mathrm{L}$

a relación cultura/sociedad-conocimiento, no se da de manera dunidireccional ; el conocimiento científico establece una relación dialógica donde ambos, cultura y conocimiento, se influyen mutuamente. Morín (1991) plantea que la ciencia no es únicamente el producto de una dinámica histórica cultural y social, sino que en sí misma se convierte en productora y transformadora de la dinámica que la produce y transforma. Los procesos sociales y materiales del mundo contemporáneo, se encuentran vigorosamente vinculados con el desarrollo científico; en el cual juega un papel importante la construcción de sistemas conceptuales que facilitan avanzar en este desarrollo.

En la actualidad, el fenómeno de la globalización y el avance de la tecnología y la informática, están generando una serie de transformaciones que ocasionan, entre otras cosas, que la construcción terminológica en los países de habla hispana se vea amenazada por la importación de tecnologías y con ello la importación de terminología de los países desarrollados. Pero, a partir de la década de los ochenta se ha venido tomando conciencia de esta situación, que

${ }^{1}$ Docente Departamento de Trabajo Social, Universidad de Antioquia, Medellín, Colombia. 
aparte de profundizar la dependencia cultural y económica de otros países, va en serio detrimento la integridad de nuestro idioma castellano, permitiendo realizar investigaciones de corte terminológico que apuntan a afianzar y fortalecer nuestro idioma.

Específicamente, el Trabajo Social en Colombia, carece de instrumentos lingüísticos que identifiquen las diferentes variantes lexicales y dialectales que surgen en el componente morfosintáctico y semántico de la lengua. Las actividades profesionales, académicas e investigativas del Trabajo Social, se realizan en ámbitos interdisciplinarios que requieren especial preparación epistemológica, investigativa y metodológica. Esto confirma la necesidad de emprender procesos investigativos de corte terminológico y documental que permitan agilizar los procesos comunicativos, afianzar el desarrollo de la profesión y fortalecerla como un espacio del conocimiento. La terminología y la documentación, pueden aportar al desarrollo de lenguajes y vocabularios especializados.

En Trabajo Social es necesario favorecer el crecimiento crítico y reflexivo, la profesión no puede encasillarse en la praxis si lo que busca es un desarrollo científico. Es ahí donde estas disciplinas pueden realizar un aporte significativo al Trabajo Social, facilitando la transferencia de información a partir de la producción de conocimiento especializado. Lo que se pretende con esta reflexión es ahondar un poco en el aporte que la terminología y la documentación pueden hacer a las demás disciplinas, concretamente al Trabajo Social.

Para cumplir el objetivo de este trabajo, que es el de reflexionar acerca de los aportes que la Terminología y la Documentación hacen a las diferentes disciplinas, específicamente a Trabajo Social, a través de sus herramientas especializadas; se hizo necesario estructurar el texto por temas conectados entre sí.

En su orden, se empieza por dar unas ideas generales acerca de la investigación, el conocimiento, la información y su contextualización en Trabajo Social; luego se trabaja la Terminología y la Documentación como disciplinas de gran importancia para el desarrollo del conocimiento, también aborda los lenguajes especializados como un producto de éstas, en esta parte da una breve explicación de los textos especializados y tres ejemplos de ellos (la base de datos, los diccionarios especializados y el tesauro); y finalmente, se hace una reflexión acerca de cómo la Terminología y la Documentación, a través de sus instrumentos, contribuyen a fortalecer el conocimiento. 
Este trabajo surge a partir de la experiencia que tuve en la pasantía del "Tesauro colombiano de Familia y Género", 2000. La investigación fue desarrollada por un equipo interdisciplinario integrado por profesionales de Trabajo Social y Bibliotecología; lo cual me aportó no solo el aprendizaje teórico-práctico a partir de lo construido, sino también una serie de elementos que contribuirán a la formación de mi perfil como profesional.

\section{La investigación, el conocimiento y la información en Trabajo Social}

Uno de los avances más importantes que ha tenido Trabajo Social, tanto a nivel teórico como de intervención, ha sido el proceso de reconceptualización; el cual ha aportado de manera significativa a un modelo propio de desarrollo de la profesión. Este proceso ha permitido reconfigurar el aspecto operativo y metodológico de ésta, realizando a la par avances teóricos y conceptuales. La investigación, punto crucial del desarrollo científico en las ciencias humanas y específicamente en Trabajo Social, debe articularse la praxis, eje del conocimiento empírico, con un desarrollo conceptual que se argumente con teorías y producciones propias de la disciplina.

Históricamente, el Trabajo Social se ha caracterizado por ela influencia de diferentes disciplinas pertenecientes al campo de las Ciencias Sociales y Humanas, con la perspectiva de orientar su vocación hacia la acción desde diversos enfoques como son: los paliativos, asistencialistas, transformadores con orientación crítica de la estructura social, democráticos, humanistas y participativos. Estos han configurado diferentes horizontes de actuación profesional hacia el desarrollo integral social y humano en contextos sociopolíticos disímiles.

En el amplio espectro de la intervención profesional de los Trabajadores Sociales, la investigación se convierte en un recurso insustituible para gestar proyectos de desarrollo humano y social, para producir procesos de seguimiento a los mismos y para conocer su impacto mediante la comprensión de las interacciones humanas y la explicación de los procesos sociales. En la actualidad es impensable la pertinencia y el impacto de la gestión social al margen de procesos investigativos (Gartner, 2000). 
La necesidad de tener actitud de indagación -investigativa- permanente en el desarrollo mismo de la intervención, el hecho de pensar la investigación como parte del desarrollo de la disciplina, es justamente poner en juego la construcción del conocimiento al servicio de ésta y de los sectores sociales con los cuales trabajamos.

Hasta finales del siglo XVII, un reducido grupo de personas podrían aspirar a dominar lo más importante del conocimiento acumulado por la humanidad. La enciclopedia de Diderot y Alambert es muestra de ello. Hoy es evidente que el conocimiento ha pasado la esfera de lo totalizable y de lo inabarcable; la información que diariamente se produce en los diferentes campos del conocimiento, es tal, que aun el estar al tanto de los avances sobre un tema específico, y más aún, acceder a un conocimiento que permanentemente se actualiza, es tarea que no puede asumir una sola persona. El conocimiento lleva consigo, además del proceso informativo y de la información misma, la adquisición mental por el estudio y la experiencia. El conocimiento se funda en un discurso, es decir, en unos actos informativos previos.

Coll-Vinent (1990) señala que la información está menos estructurada que el conocimiento; y suele estar constituida por medios aislados no relacionados del todo, cuando estas se ordenan y se asocian a algunas estructuras de entendimiento, se habla entonces de conocimiento. De esta manera se puede ver que existe una continuidad pero también una separación entre información y conocimiento. Asimismo, el término información presenta innumerables significaciones, dependiendo del uso del contexto, y de cuantos factores estén implicados por ella. Se ha dicho que la información habrá que añadirla a esa lista de vocablos indefinibles: espacio, tiempo, energía, etc.

De acuerdo al Glosario de Bibliotecología y Ciencias de la Información de "American Library Asociation", la información es definida como " todas las ideas, hechos y trabajos imaginativos de la mente que se han comunicado, registrado, publicado y/o propagado formal o informalmente, en cualquier forma." Esta definición lleva consigo la idea de que la información es acción de informar, en consecuencia, siempre está ordenada por alguien y para unos destinatarios, requiere para ello medios y lenguajes y se sirve de un proceso de transmisión.

Hoy en día se concibe la información como un recurso del conocimiento y del quehacer humano. De acuerdo a Coll-Vinent (1990), 
la sociedad actual está configurando sus alternativas de recambio por lo que a los recursos se refiere, con base a la utilización de la información, a todos los niveles; antes que la manipulación material de los recursos energéticos propiamente dichos. En este estado de cosas, se subraya el valor que va adquiriendo la información, y muy especialmente la que tiene carácter científico y técnico, y eesto significa poder en el más amplio sentido de la palabra. Es decir, que el que carece de información carece de poder y de capacidad de acción ya sea proyectiva o ejecutiva (Coll-Vinent,1990).

Ninguna realización científica se produce a partir de cero, cualquier conocimiento se basa en un saber previo y en un cierto lenguaje preconstituido. Este comportamiento en el encadenamiento de nuevos conocimientos y hallazgos científicos, define el carácter acumulativo básico de la ciencia que afecta a todas las disciplinas. Este carácter acumulativo es posible gracias a la instrumentación documental que le sirve de fuente y palanca a la vez. Es impensable imaginar el desarrollo de la ciencia y la tecnología sin los actuales sistemas de información y documentación. Esta vinculación no es una mera instrumentalidad: se trata de un nuevo elemento de la metodología científica, considerada tanto en su quehacer creativo como en su producción institucional más amplia. La característica documental forma parte de la epistemología científica, en otras palabras, la documentación es necesaria para la ciencia.

\section{La documentación}

La documentación es el proceso de comunicación, por medio del cual el individuo recibe las referencias de unos objetos debidamente tratados, expresa o presuntamente pedidos, relativos a un determinado dominio de la actividad social que el individuo desempeña, y que le hace competente para el ejercicio de esa actividad, cuando atinadamente lo maneja. Es una disciplina relativamente joven, que tiene como objeto de estudio el flujo de la transferencia de información; fenómeno sistémico que supone los procesos de: seleccionar, adquirir, analizar y difundir documentos; entendiendo por documento, no solo los medios impresos, sino todo aquello que contenga y proporcione información que se puede comunicar, que tenga fácil acceso y que sea permanente para luego ponerlos al servicio de una comunidad ya delimitada. 
Este proceso actúa como un sistema regulador de la información, la recoge desde sus fuentes originales, la interpreta y la canaliza, lo que crea información y con ello se inicia nuevamente el ciclo. La transmisión de la información es el eje y el primer objeto de la documentación. Su participación es informativa en tanto que le presta la fuente, la comunicación y la nueva fuente que de todo el proceso se derive en un incesante e interminable proceso informativo.

La documentación rige así todo el proceso intercomunicativo entre los científicos y el conocimiento. Es una disciplina normativa proveedora de pautas para la consecución efectiva y eficaz de los conocimientos científicos. Esta no se ha separado del todo de un tronco común por el procedimiento habitual de la especialización y fragmentación del conocimiento, sino que mantiene como ciencia informativa de la ciencia, una presencia significativa en todos y cada uno de los saberes particulares. La función informadora de la documentación, corresponde a la etapa preliminar de la investigación terminológica; el vasto campo de la información y de la documentación es un componente imprescindible de la terminología.

\section{La terminología}

Las prioridades y los métodos de trabajo de cualquier disciplina, aparecen cuando su necesidad se pone de manifiesto, y se desarrollan en mayor o menor grado en la medida en que responden a necesidades sociales evidentes. El análisis de la organización de las sociedades y de sus ideologías representativas permite establecer las causas de la aparición de la terminología y permite también explicar la importancia que hoy día ha adquirido en la mayoría de países avanzados.

La terminología es una disciplina relativamente joven dentro de las ciencias lingüísticas aplicadas, su fundamentación teórica apenas empezó a consolidarse concretamente entre los años sesenta y setenta. Para definirla, es necesario antes establecer su relación y su delimitación con el lenguaje común y el tecnolecto. Fedor De Diego y Boroni (1983) plantea, que el lenguaje común es una forma del idioma que tiene validez en todo el territorio en donde se habla ese idioma y que es entendible por todos los que pertenecen a ese grupo lingüístico, se usa para el intercambio de pensamientos de índole general, es decir, sin una orientación específica dada por algún 
campo del saber. Define el tecnolecto como el conjunto de formas de aparición del idioma, que sirve para la comunicación especializada entre expertos de las más diversas áreas. La terminología, es definida como el vocabulario utilizado en el tecnolecto de algún campo del saber especializado, es decir, la cantidad de denominaciones (términos que se utilizan en un campo del saber especializado en forma diferente del usado en el lenguaje común) (Fedor De Diego y Boroni, 1983). En otras palabras, se puede deducir de esta definición, que los términos son las piedras fundamentales de cualquier tecnolecto.

Estos tres fenómenos lingüísticos, presentan correlaciones donde las delimitaciones no se dan fácilmente: el tecnolecto no puede existir sin un lenguaje común, sin embargo, éste sí puede funcionar sin necesidad de incluir elementos de tecnolectos. De otro lado, la aproximación terminológica en los tecnolectos, parte exclusivamente de la noción precisa y objetiva asignándole una sola denominación. El término no admite sinónimos ni plurivalencias de ningún tipo, y la normalización de la terminología es una necesidad para llegar a una comunicación inequívoca y sin ambigüedades.

La terminología se considera como una materia de encrucijada entre la lingüística, la ciencia cognitiva, la comunicación y la informática. Su carácter interdisciplinario se fundamenta en la interseccsión que sugiere el proceso terminológico entre éstas disciplinas.

Es considerada una transdisciplina puesto que no hay ninguna materia especializada sin terminología, no se puede comunicar la especialidad sin la misma y no hay terminología sin materia especializada.

Es una interdisciplina, puesto que como todas las materias científicas interdisciplinarias, es una materia que se define en relación con otras materias, de las que toma prestados un conjunto específico de conceptos. Una interdisciplina no define un campo de estudio como una adición lineal de los conceptos procedentes de las disciplinas que la conforman, sino que selecciona de las mismas un determinado número de conceptos y elementos y posteriormente elabora a partir de esos conceptos un objeto y un campo propio.

El objeto de la terminología enfocada como una disciplina y analizada desde el punto de vista teórico, es el de cualquier materia científica: Explicar las unidades terminológicas y su uso por parte de los especialistas en sus diferentes niveles y circunstancias de especialización. 


\section{Relación entre documentación y terminología}

La terminología y la documentación son dos disciplinas que se sirven mutuamente, no es posible concebir el desarrollo de una sin el apoyo de la otra; ambas tienen un carácter interdisciplinario ya que se nutren de otras ciencias y pueden aplicarse a cualquier área del conocimiento. El vínculo entre ambas es doble: las mismas precisan para sus herramientas de trabajo términos bien definidos y estructurados; la terminología se aprovecha de estas herramientas documentales para sus fines, esto es, que con frecuencia se parte de la ya existente estructuración de un campo del saber en clasificaciones para desarrollarla y convertirla en un verdadero sistema de conceptos.

La función documental en terminología debe referirse tanto al fondo como a la forma. El proceso terminológico parte del concepto para desembocar en el término. Entonces la documentación terminológica tiene una función doble, sea un papel a la vez de la información y la formación, y un papel lingüístico. La documentación para poner la información al servicio de los usuarios, requiere de unas buenas herramientas terminológicas (como los tesauros y las listas de clasificaciones temáticas). De igual manera una de las bases más importantes de la terminología es la documentación, ya que antes de desarrollar un trabajo terminológico, se debe hacer una exhaustiva selección y análisis bibliográfico correspondiente al área en la cual se pretenda desarrollar el corpus temático, que según Barite (1997) es el conjunto de fuentes orales y escritas que son utilizadas en un trabajo terminológico especializado.

Rondeau (1980) señala que la terminología y la documentación son indisolubles una de otra, en el sentido de que es imposible hacer terminología sin tener acceso a una abundante documentación especializada. La terminología necesita de la documentación para conocer la estructura conceptual de un ámbito, recopilar los términos y conocer los contextos de uso de los términos; la documentación necesita de la terminologíia para indizar los documentos y construir lenguajes especializados. 


\section{Los lenguajes especializados}

Antes de definir que es un lenguaje especializado, es importante tener una idea general de lo que es el lenguaje. Para Arellano y otros (2000), el lenguaje es la capacidad humana que le permite al hombre comunicarse por medio de la palabra o la escritura, utilizando códigos comunes entre una comunidad; el hablante los utiliza y selecciona de acuerdo a sus necesidades de comunicación. La información y el desarrollo de los lenguajes especializados, constituyen una de las materias primas para la generación del conocimiento. La capacidad para generar eficiente y oportunamente este recurso y sobre todo su procesamiento social e individual para que se convierta en conocimiento nuevo, permite no soo mantenerse al día con respecto a los vertiginosos cambios sociales, culturales y tecnológicos, sino incorporarlos a la vida práctica generando así una mejor calidad de vida (Cabré, 1993).

Todo trabajo terminológico es por excelencia un lenguaje especializado, ya que sus estudios se realizan a partir de la investigación de los términos y conceptos que se utilizan en un área específica. La ausencia de una terminología especializada en cualquier área genera serios problemas de comunicación y de transferencia de conocimientos, produciendo una carencia al momento de tratar de unificar conceptos y criterios. El inicio de un trabajo terminológico, por sus características específicas lleva a los especialistas a reflexionar sobre los fundamentos teóricos, epistemológicos y el quehacer profesional (Arellano y otros, 2000).

Un lenguaje especializado es el conjunto de todos los recursos lingüísticos que se utilizan en un ámbito comunicativo delimitable para el especialista, lo que garantiza la comprensión entre las personas que trabajan en este ámbito. Arellano y otros (2000) afirman que el lenguaje de especialidad es la forma práctica elegida y desarrollada por los científicos y técnicos para remediar los problemas de comunicación que existen entre ellos, la difusión del conocimiento en un área especializada, requiere de un estudio del lenguaje utilizado para permitir que los conceptos transmitidos sean interpretados en el mismo sentido en que fueron emitidos, sin embargo, podríamos afirmar que el lenguaje de especialidad no es estático en cuanto a que con su uso se puede convertir en un lenguaje común.

La existencia del lenguaje común es indispensable para el lenguaje especializado, este toma los términos y los contextualiza de 
acuerdo a unos parámetros ya establecidos; no obstante, el lenguaje común puede tomar términos del lenguaje especializado sin que estos sean necesarios para su permanencia. Por otro lado, los lenguajes de especialidad son códigos lingüísticos que están semánticamente controlados; además, permiten la entrada de nuevas unidades terminológicas haciendo un uso moderado de los sinónimos, ya que entre menos se hagan uso de estos, es mucho más efectivo el proceso de comunicación. Como producto de estos lenguajes de especialidad surgen los textos especializados con el objetivo de comunicar y difundir la información especializada de determinada área del saber.

\section{Los textos especializados}

Los textos especializados suelen ser de carácter referencial, y por lo tanto, presentan aquellos recursos sintácticos y textuales que sirven para dar una información lo más objetivamente posible y cuanto más despersonalizada mejor. Estos hacen parte de la documentación especializada (la documentación que tiene una relación más estrecha con las actividades del terminólogo y que permite la construcción de vocabularios más técnico-científicos), que se desprende a su vez (al igual que la documentación general y la documentación industrial) de la documentación escrita De acuerdo a Heroux (1983), esta sirve de base para el establecimiento del corpus que tiene dos facetas: el corpus de análisis, que será examinado para fines de análisis terminológico; y el corpus de referencia, que está utilizado a la vez en la fase preparatoria de los trabajos y durante la investigación.

Los textos especializados tienen un carácter complejo; a nivel lingüístico, porque han sido construidos a partir de la gramática de una lengua; a nivel pragmático, porque el uso que puede hacerse de ellos es variado y multidimensional; y a nivel socio-lingüístico, porque una lengua es un sistema de comunicación social que ocupa un lugar determinado en la sociedad que la utiliza, bien sea por la relación que mantiene con otras lenguas con las que comparte con más o menos fluidez un mismo espacio, o por la relación que tiene con lenguas de otras sociedades.

El desarrollo del lenguaje y de textos especializados, es un método práctico para enfrentar los problemas de comunicación que surgen entre las diferentes disciplinas; además pueden contribuir a 
estructurar un sistema de conceptos propios y a aclarar las principales nociones de la profesión. En este sentido, se pueden identificar, en lo que se refiere a la terminología con el sector de "información refinada", algunos textos especializados como las bases de datos, los diccionarios especializados y los tesauros, entre otros. Estos textos hacen parte del lenguaje artificial o convencional que es un lenguaje estructurado con el propósito de recuperar la información y el conocimiento.

\section{La Base de Datos}

Son una serie de archivos enlazados entre sí, diseñados para facilitar la recuperación de la información (Coll-Vinent, 1990) e identificar las principales tendencias teóricas y metodológicas presentes en los procesos investigativos. Esta agrupa la información de acuerdo a criterios establecidos. Marban (1987) indica que es una colección de datos relacionados entre sí de cierta forma y organizados para que sea posible encontrar en ella la información que se desea.

En el caso de la información especializada, la terminología empleada será también especializada, lo cual inf luye sobre el vocabulario que se emplee, ya sea que se usen tesauros o vocabularios controlados.

Se trata de una investigación documental con profundos nexos con lo empírico, por cuanto parte de los hallazgos que en torno a la situación objeto de análisis han hecho los estudios previos y recupera la experiencia acumulada por los investigadores con trayectoria en el campo específico del saber al cual se refiere.

\section{El Diccionario especializado}

La publicación de diccionarios especializados, ofrece la posibilidad de dar un tratamiento adecuado al lenguaje técnico y de evitar los barbarismos que se utilizan con frecuencia (Alpizar, 1997). Más aún cuando se presentan ediciones renovadas que dan cuenta de la evolución y progreso de cada especialidad. Alpizar (1997) también plantea que los diccionarios generales no pueden contener ni una íntima parte del vocabulario de la ciencia y de la técnica, por diversas razones que van desde la velocidad con que en la vida moderna se suceden los inventos e innovaciones, hasta la imprescindible sencillez con que una obra dedicada a un público 
no especializado, y debe definir conceptos que muchas veces son de difícil comprensión para los no iniciados en determinada rama del conocimiento.

La importancia de elaborar diccionarios científicos o técnicos radica precisamente en la imposibilidad que tiene el diccionario general de dar una respuesta adecuada al movimiento terminológico mundial actual. Su estructura está conformada por dos elementos: la macroestructura, que es el conjunto de las partes principales que componen la obra, (parte introductoria, cuerpo de la obra y anexos); una microestrucutura, que es el conjunto de elementos y la disposición interna que presenta cada uno de los artículos que componen la obra lexicográfica (lema, información gramatical, definición y notas de alcance).

El aporte que este tipo de obras hace al quehacer científicoinvestigativo; es considerable: ellas se convierten en un factor de precisión en la comunicación científica, contribuyen en la aclaración de dudas y, en cierta medida, a la eliminación de disparidades y la unificación de criterios.

\section{El Tesauro}

Tiene su origen en el control terminológico de las denominaciones de un área de especialidad, correspondientes a este control su finalidad (Cardona, 1999). Es una compilación de palabras y frases que ponen de manifiesto sinónimos, relaciones jerárquicas y dependencias cuya función es suministrar un vocabulario estandarizado para el almacenamiento de información en los sistemas de recuperación. Coll-Vinent (1990) lo define como un conjunto organizado de descriptores seleccionados en razón de su aptitud para poner en evidencia el contenido de los documentos de un campo determinado del saber. Y una lista organizada de términos extraídos de un vocabulario especializado, ordenados de suerte que faciliten la selección de descriptores.

Debido a que el tesauro es fundamentalmente un instrumento de recuperación de información contenidas en los documentos de un determinado centro de documentación o una base de datos, su estructura es concebida para que cualquier usuario pueda realizar con el una búsqueda sin esfuerzo y en poco tiempo, lo cual quiere decir que su manejo ha de ser fácil y sin complicaciones (CollVinent, 1990). ${ }^{3}$ 
Su estructura generalmente consta de tres partes: la primera, llamada estructura de conjunto, constituida por los grandes epígrafes del tema sobre el que versa el tesauro, que suele ser casi siempre una parcela específica de una ciencia o una técnica determinada; una segunda parte, sistemática, que explícita máximamente el contenido de los temas enunciados en los epígrafes de la estructura de conjunto y está integrada por nuevos epígrafes cada vez más específicos; y la tercera, que es un indice de descriptores alfabéticamente ordenado, cada descriptor va seguido de un código que remite al índice sistemático donde se puede encontrar una información más amplia y más detallada.

Cardona (1999) señala que el tesauro es utilizado como herramienta de control terminológico, como un instrumento que da cuenta del lenguaje especializado o como un lenguaje de indización y recuperación de información. Tanto las bases de datos, los diccionarios especializados y los tesauros; son herramientas documentales que contribuyen a fortalecer sistemas conceptuales específicos que permitan el desarrollo práctico y unificado en la evolución de cualquier ciencia o disciplina; más ahora, cuando se ha convertido en un imperativo para éstas la claridad conceptual de los términos que constituyen la base del conocimiento aplicado al trabajo como profesionales expertos en áreas específicas.

\section{Aporte de los textos especializados al Trabajo Social}

A continuación haré una breve reflexión acerca de los aportes que la Terminología y la Documentación pueden hacer al Trabajo Social.

Según la aproximación tradicional, en Trabajo Social como disciplina, se ha dado una centralización llevada al extremo hacia los aspectos prácticos y específicos, Trabajo Social ha sido una profesión marcada por el asistencialismo que privilegia las acciones específicas y de corto alcance sobre el establecimiento de concepciones epistemológicas y teóricas que promueven el crecimiento hacia opciones más generales del conocimiento. En términos concretos, ha primado la aplicación de técnicas pragmáticas sin un trasfondo y fundamento crítico porqué su utilización.

La experiencia ha sido colocada por encima de la crítica, lo que crea impedimentos al desarrollo de una opción científica. Simón 
Castillejos y Juan Barriex, en su libro "Metodología y método del Trabajo Social”, afirmaron que, según Bachelard, a la experiencia se le asumía desde dimensiones de añoranza y nostalgia, no se tenía en cuenta un carácter de objetivación, sino que lo realizado mediante la praxis es lo que se tomaba como válido y real. La experiencia concreta no se asumía como un antecedente profesional que podía enriquecer desarrollos nuevos y creativos; sino que se le tomaba como reproducción idéntica, sin importar si las circunstancias eran distintas en el espacio y el lugar. Con esta problemática no había crecimiento a nivel conceptual, ni superación de errores (Arrelano y otros, 2000).

Malcom Payne, en su libro Teorías Contemporáneas de Trabajo Social, presentó el desarrollo de las teorías en un contexto histórico, social, político y cultural; relacionó la interacción de tres agentes importantes: asistente social, cliente social, y medio en el cual actúaban los dos anteriores. Luego planteó la utilización de la teoría y la práctica en el quehacer profesional contextualizando con otras teorías que han sido soportes o complemento de la práctica y las críticas que surgían frente a ella, finalmente hizo sugerencias para unir la teoría y la práctica especialmente desde la investigación.

De acuerdo a Coria y otros (1999), lo anterior sustenta que la investigación debe cobrar mayor importancia tanto en la formación como en la práctica del Trabajo Social, tiene que buscar mecanismos que le ayuden a resolver su relación históricamente conflictiva con el conocimiento, para resignificar la disciplina y parapara fundar mejores intervenciones. Para emprender un proceso de resignificación de la disciplina, es necesario buscar mecanismos que permitan crear una unidad de criterios necesarios para establecer un equilibrio entre la teoría y la práctica; de ahí la importancia de construir textos especializados como las bases de datos, los diccionarios especializados, y los tesauros; que pueden permitir un desarrollo epistemológico de la profesión y al mismo tiempo facilitar el intercambio de la información en el marco de un lenguaje controlado.

Cada área del saber lleva implícito un cúmulo de conocimiento que la sustenta. Es decir, cada disciplina presenta conceptos y conocimientos específicos que la caracterizan. Para su adecuada información y divulgación, es necesario valerse de mecanismos que permitan hacerlo con eficiencia. Lo anterior fundamenta la importancia de la comunicación especializada. Cuervo (1999) indica que los grandes cambios que se están generando el nivel mundial y del 
continente Latinoamericano han afectado y afectarán las políticas sociales y el quehacer de los profesionales. Específicamente el Trabajo Social se está enfrentando a la realidad de tener teorías muy difusas que no le han permitido analizar las situaciones tan complejas que se presentan en el trabajo de campo, llevándolo a tener que apoyarse en lo interdisciplinario y por ende valiéndose de los aportes de otras ciencias. La profesión históricamente no ha logrado derivar teoría de la experiencia práctica, los intentos de sistematización de las experiencias no han trascendido en la producción de conocimiento.

La base conceptual del Trabajo Social tuvo su origen en Europa y a través de la historia fue copiada primero en Norte América y más tarde en América Latina. Además de los conceptos también fueron adaptados los métodos y técnicas sin tener en cuenta las diferencias culturales, lo que puso en evidencia una descontextualización, dado que tanto las problemáticas presentadas como las dinámicas institucionales y los recursos existentes, sugieren una intervención acorde a las condiciones del medio. Para que los efectos producidos sean los esperados, es necesario indagar por la evolución de conceptos que permita contextualizarlos de acuerdo a las condiciones políticas sociales y económicas de cada región.

Arellano y otros (2000) plantea que en la profesión se ha producido un conocimiento generalista que se encuentra ligado a una concepción empirista de la realidad, dicho empirismo va directamente en contra del pensamiento y la acción científica. La posición empirista no hace cuestionamientos, no se pregunta acerca del porque de los procesos, no busca su comprensión desde el conocer, que es en últimas lo que pretende la aproximación científica. La rigurosidad científica es una falencia del Trabajo Social. Al interior de nuestra disciplina, se maneja un discurso sin fundamentos con base en el cual se concretan acciones en donde prevalecen la motivación y la conveniencia de la ideología de turno. Esto pone en evidencia una inclinación por asumir posiciones facilistas para evadir la responsabilidad de una intervención fundamentada en el conocimiento científico y en el rigor de sus planteamientos.

En Trabajo Social existe una idealización de lo social que dificulta comprender la realidad actual. La visión y el imaginario de Trabajo Social están enmarcados por interpretaciones restrictivas del proceso de modernidad y por la asunción de modelos sociales clásicos que definen su identidad profesional. La actitud general 
sobre esta situación es más de incertidumbre que de indagación y eso dificulta la búsqueda de nuevas formas de comprender e intervenir en las sociedades complejas de hoy ((Vélez, 2000).

Lo anterior sustenta la necesidad de establecer mecanismos que promuevan la realización de investigaciones para consolidar un cuerpo teórico acorde a la situación latinoamericana, específicamente a la colombiana; y para reconceptualizar nuestro quehacer profesional, buscando adquirir una capacidad de percepción integral de lo social, valiéndonos de las herramientas terminológicas y documentales que facilitan mayor claridad y precisión conceptual respecto al lenguaje utilizado.

Por esta razón, considero importante y de gran utilidad para el Trabajo Social estos procesos investigativos que se han dado o que se vienen dando dentro de la línea investigativa dei CISH (Base de Datos "Guía bibliográfica de Familia y Género", Diccionario especializado de Trabajo Social y Tesauro de Familia y Género), puesto que además de contribuir al fortalecimiento de la investigación como medio para adquirir y producir conocimiento, favorecen el crecimiento crítico y reflexivo de la profesión, buscando un desarrollo científico que trascienda la praxis, facilitando la transferencia de información para favorecer el crecimiento y la producción de conocimiento especializado.

Leal (2000) señala que la ausencia en Colombia de este tipo de herramientas especializadas, produce una carencia de unidad de criterios en conceptos que tradicionalmente se vienen incorporando en el espacio profesional, lo cual amerita profundizar e investigar acerca de cuales son los términos utilizados y que alcance tienen, para facilitar los procesos de transferencia de la información y el conocimiento de realidades en las que se interviene.

La palabra es la herramienta más importante que ocupa puesto especial en cada organismo social, de cuya trascendencia no puede pasar desapercibido el Trabajo Social. Teniendo en cuenta que la consolidación de comunidades académicas basadas en la actividad investigativo que trasciendan los límites de lo local es un imperativo para el desarrollo del conocimiento en la sociedad contemporánea, se hace necesario la implementación de estrategias que estimulen la capacidad de interacción y de cooperación entre investigadores. La creación de un sistema conceptual facilita el fortalecimiento de comunidades académicas de Trabajo Social, que es una condición indispensable para el desarrollo de los currículos y de la profesión. 
Estos trabajos pueden generar una construcción de un instrumento lingüístico que le permita identificar las diferentes variables lexicales y dialectales que surgen en el componente morfosintáctico y semántico de la lengua; además pueden permitir la adquisición de una preparación especial, epistemológica, investigativa y metodológica para desempeñar las diferentes actividades profesionales, académicas e investigativas, agilizando los procesos comunicativos y afianzando su desarrollo bien como profesión o como espacio del conocimiento.

Resignificar el Trabajo Social de hoy, colocándolo en un horizonte que tenga como fundamento la rigurosa y compleja comprensión de lo social, eliminando las tensiones entre teoría y práctica; es uno de los desafíos centrales de la profesión en el siglo XXI. Trabajo Social debe pensarse desde afuera y desde adentro: desde los procesos que le son propios y en los cuales se inserta; y desde los sistemas y el mundo de la vida para construir desde ahí, lenguajes que tengan la capacidad de cambiar y develar las voces de la esperanza (Vélez, 2000).

\section{Conclusiones}

- Las herramientas de corte terminológico o documental, pueden contribuir a fortalecer sistemas conceptuales específicos que permitan el desarrollo práctico y unificado en la evolución de cualquier ciencia o disciplina; puesto que además de contribuir al fortalecimiento de la investigación como medio para adquirir y producir conocimiento, favorece el crecimiento crítico y reflexivo de la profesión, buscando un desarrollo científico que trascienda la praxis, facilitando la transferencia de información, para favorecer el crecimiento de la producción de conocimiento especializado.

- El trabajo interdisciplinario, representa un diálogo entre saberes que dinamizan los procesos de cada uno en su interior, porque demanda un esfuerzo por realizar precisiones teóricas desde cada mirada.

- La colaboración entre expertos de las diferentes especialidades y los linguistas terminólogos, es condición necesaria para 
enfrentar los peligros que acechan la comunicación especializada en nuestra época tecnológica.

- Teniendo en cuenta que la consolidación de las comunidades académicas basadas en la actividad investigativa que trasciendan los límites de lo local es un imperativo para el desarrollo del conocimiento en la sociedad contemporánea, se hace necesario la implementación de mecanismos que estimulen la capacidad de interacción y de cooperación entre investigadores. La creación de un sistema conceptual facilita el fortalecimiento de comunidades académicas de Trabajo Social que es una condición para el desarrollo de los currículos y de la profesión.

- El desarrollo del lenguaje y de textos especializados, es un método práctico para solucionar los problemas de comunicación que surgen entre las diferentes disciplinas, además contribuye a estructurar sistemas de conceptos propios y a aclarar las principales nociones de la profesión.

\section{Bibliografía}

Alpizar, Rodolfo (1997). ¿̇Cómo hacer un diccionario científico técnico? Ed. Memphis, Buenos Aires.

Arellano, José Fernando; Cano, Alejandro y Otros (2000). Diccionario especializado de Trabajo Social, informe de avance de investigación. Medellín, Universidad de Antioquia,CISH.

Barite, Mario (1997). Glosario sobre organización y representación del conocimiento: clasificación, indagación, terminología. Montevideo: Comisión sectorial de investigación científica y Escuela universitaria de Bibliotecología y Ciencias Afines.

Cabré, María Teresa (1997). La Terminología: marco teórico, metodología y consecuencias prácticas. Universidad de Antioquia, Curso de Terminología, 4-7 de noviembre. 
Cabré, María Teresa (1993). La Terminologia: teoría, metodología y aplicaciones. Barcelona: Ed. Antártida.

Cardona, Bertha Nelly (1997). Manual metodológico para la elaboración del Tesauro Agrícola colombiano. Medellín.

Cardona, Bertha Nelly (1992). Guía metodológica para la elaboración de un tesauro. Santafé de Bogotá: SIDES.

Cardona, Bertha Nelly (1999). Terminología y Documentación. I Jornadas Iberoamericanas de Terminología. Santafé de Bogotá, septiembre 13-17.

Cerda, Hugo (1994). La investigación total. Santafé de Bogotá: Ed. Magisterio.

Coll-Vinent, Roberto y Bernal, F. (1990). Curso de Documentación (2da, ed.). Madrid: Ed. Dossat, S.A.

Coria, Adela y Otros (1999). Currículum, investigación y Trabajo Social. Buenos Aires: Ed. Espacio.

Cuervo, Gloria (2000). Diccionario especializado de Trabajo Social: un aporte a la profesión desde la metodología. Revista Colombiana de Trabajo Social, 14.

Fedor De Diego, Alicia y Boroni, Michele (1984). Actas del primer seminario nacional de Terminología. Caracas: Ed. Universidad Simón Bolivar.

Gartner, Lorena (2000). La investigación en la formación de Trabajo Social: su proyección y condiciones de posibilidad. Revista Colombiana de Trabajo Social, 14.

Marban, Rocío (1987). Diseño de bases de datos. OEA: ILAITI.

Morin, Edgar (1991). El Método, las ideas. Madrid: Ediciones Cátedra.

Noguera, Nuria (1999). Documentación científica y nuevas tecnologias de la Información. España: Ed. Pirámide. 
Quintero, Angela María (1999). Guía temática de Familia y Género. Medellín: Universidad de Antioquia, CISH, FBH.

Rondeau, Guy (1980). Terminologie et documentation. META, 25 (1). Marzo.

Valencia, Adriana y Cardona, E. (1999). Lenguajes de especialidad (inglés) como herramientas de transferencia del conocimiento. IJornadas Iberoamericanas de Terminología. Santafé de Bogotá, septiembre 13-17.

Vélez, Olga L (2000). Perspectivas del Trabajo Social en el siglo XIX. Revista Colombiana de Trabajo Social , 14. 